\title{
Condições de Trabalho e de Saúde de Trabalhadores da Saúde Hospitalar
}

\section{Working and Health Conditions of Hospital Health Workers}

\section{RESUMO}

Objetivo: Caracterizar as condições gerais de trabalho e de saúde dos profissionais de saúde hospitalar. Metodologia: Estudo epidemiológico de corte transversal, com amostra de trabalhadores da saúde hospitalar em município de médio porte. Foi utilizado um instrumento de coleta composto por blocos de questões e aplicado no local de trabalho. Após a coleta, procedeu-se com análise estratificada por função do trabalhador, assistencial ou administrativa. Por fim aplicou-se o teste estatístico para avaliar a diferença entre os grupos. Resultados: O perfil sociodemográfico da população estudada aponta para uma maioria do sexo feminino, entre 31 e 40 anos, casados, com cor da pele autodeclarada preta/parda, residentes na Cidade de Jequié e com filhos. Grande parte dos profissionais trabalha há 11 anos ou mais na profissão, com carga horária de 30 horas semanais, além de exercerem outra atividade remunerada e, expressiva maioria, desempenha atividades domésticas. Parcela considerável dos participantes apreciava o seu estado atual de saúde, apesar de expressivo número de profissionais com distintas comorbidades, destacando-se a alta prevalência de sintomas osteomusculares. Conclusão: A identificação das condições gerais de trabalho e as principais doenças que afetavam esse público, em conjunto com uma proposta de inferência da relação entre tais variáveis, pode contribuir para a melhoria da compreensão da situação de saúde do trabalhador do setor de saúde hospitalar.

\section{DESCRITORES}

Saúde do Trabalhador. Condições de Trabalho. Trabalhadores da Saúde.

\begin{abstract}
Objective: to characterize the general conditions of work and health of the professionals in a hospital. Methodology: cross-sectional study, with a sample of hospital health workers. A collection instrument composed of blocks of questions was used and applied in the workplace. After collection, we proceeded with a stratified analysis by function of the worker, assistant or administrative occupations. Finally, a statistical test was applied to assess the difference between the groups. Results: the sociodemographic profile of the studied population points to a majority of females, between 31 and 40 years old, married, with self-declared skin color black/brown, residents in the town of Jequié and with children. Most of these professionals worked 11 years or more in this profession, with a workload of 30 hours a week, in addiction to fulfilling other paid activity and an expressive majority performed domestic activities. A considerable part of the sample appreciated their current health condition, although a significant amount of the professionals with various comorbidities, highlighting the high prevalence of musculoskeletal symptoms. Conclusion: the identification of the general conditions of work and major diseases that affected this population, together with the attempt to inference the relationship between such variables, can contribute to the improvement of understanding the situation of welfare of the hospital health workers.
\end{abstract}

\section{DESCRIPTIONS}

Occupational Health. Working Conditions. Health Personnel. 
A Lei Orgânica da Saúde - Lei nº 8.080/1990¹ trouxe para a realidade brasileira a definição ampliada do conceito de saúde. Nesse contexto, o trabalho ocupa lugar de destaque na promoção do bem-estar do ser humano, sendo necessário, dessa forma, o enfoque na relação homem-atividade ocupacional. Nessa perspectiva, destaca-se a Saúde do Trabalhador, um campo do saber que visa compreender as relações entre o trabalho e o processo saúde/doença; considerando-se a saúde e a doença como processos dinâmicos, estreitamente articulados com os modos de desenvolvimento produtivo da humanidade em determinado momento histórico ${ }^{2}$.

Quando se fala no processo saúde-doença do trabalhador, além da importância naturalmente concernente aos impactos no sistema de saúde, tem-se todo o prejuízo inerente ao fato de a população atingida ser a economicamente ativa e toda e qualquer alteração na economia tem consequências deletérias, tanto individual quanto coletivamente. A Organização Internacional do Trabalho (OIT) estima que os acidentes de trabalho e as doenças profissionais resultam numa perda anual de $4 \%$ no produto interno bruto (PIB) mundial, ou cerca de 2,8 bilhões de dólares, em custos diretos e indiretos de lesões e doenças ${ }^{3}$.

A globalização trouxe consigo mudanças tecnológicas, sociais e organizacionais no ambiente de trabalho e na forma como ele é executado e isso suscita em riscos emergentes e novos desafios, no que diz respeito à saúde do trabalhador. Segundo a OIT, embora alguns riscos tradicionais tenham diminuído em virtude, por exemplo, de melhorias na segurança e regulamentação, eles continuam a provocar danos inaceitáveis à saúde dos trabalhadores; ademais, existe o aumento de novas formas de doenças profissionais, que ainda não têm implementação de medidas adequadas de prevenção, proteção e contro$\mathrm{le}^{3}$.

Os trabalhadores em saúde atuam no nível da gestão ou da assistência direta ao cidadão doente ou atendido em programas de promoção da saúde, sendo que podem também atuar nos serviços de apoio diagnóstico e terapêutico, na gerência e na produção de tecnologia ${ }^{4}$. As transformações recentes na produção capitalista em nível mundial também se fizeram presentes no setor saúde, que de maneira semelhante aos outros setores da produção experimentaram não somente os efeitos do avanço da ciência e da tecnologia, mas também do acirramento da desigualdade e da injustiça social que marcam muitas nações ${ }^{4}$. No plano global, os serviços de saúde têm sofrido erosão, falta de investimentos, ajustes estruturais e cortes de financiamento, além disso, existe a alta carga de trabalho e as condições de trabalho não motivadoras, incluindo os riscos ${ }^{4}$.

Devido ao fato de os recursos humanos constituírem a base do sistema de saúde ${ }^{4}$, sendo o trabalho dos profissionais desse setor associado, direta ou indiretamente, ao cuidado de outrem, é mais do que necessário que se conheça as principais condições de saúde e doença dessa população, caracterizando o perfil de morbidade e elucidando o impacto de tais problemas na execução do seu trabalho e no seu bem-estar. Dessa forma, o objetivo desse trabalho foi caracterizar as condições gerais de trabalho e saúde dos profissionais de saúde hospitalar. 


\section{METODOLOGIA}

Trata-se de um estudo epidemiológico de corte transversal e descritivo, realizado em unidade hospitalar localizada no Município de Jequié, Região Sudoeste do Estado da Bahia, com uma população de 162.209 pessoas, estimada em $2017^{5}$. O local do estudo foi um dos principais hospitais de referência regional do interior do Estado da Bahia, fundado em 1947, possuindo mais de 200 leitos operacionais, internando nas especialidades de Clínica Médica, Clínica Cirúrgica, Clínica Neurológica, Pediatria, Psiquiatria, Neonatologia, Obstetrícia e Terapia Intensiva ${ }^{6}$.

A população de estudo foi composta por trabalhadores da saúde do referido hospital. O cálculo do tamanho da amostra foi baseado na ocorrência geral de sintomatologia musculoesquelética entre os profissionais de saúde. Como a investigação dessa ocorrência poderia ser influenciada pela questão do desenvolvimento da jornada de trabalho dos participantes, como já foi demonstrado em estudos na área ${ }^{7,8}$. O cálculo foi conduzido pelo sistema openepi (www.openepi.com) e foi considerado a prevalência geral de dor musculoesquelética de 75,0\%; população de 643 profissionais de saúde na referida unidade, limite de confiança de $5 \%$ e efeito de desenho igual a um. O tamanho final da amostra foi de 200 participantes. A partir da lista de profissionais de saúde da referida unidade hospitalar foi realizado um sorteio simples.

$O$ instrumento de pesquisa utilizado foi um questionário composto por nove blocos de informação, que incluíam: informações sociodemográficas, características ocupacionais, aspectos psicossociais do trabalho, ativida- des domésticas, atividades de lazer, hábitos de fumar e consumo de bebidas alcóolicas, aspectos gerais de saúde, sintomas osteomusculares, condições de vida e acidentes de trabalho.

A coleta de dados ocorreu no respectivo cenário de estudo, sendo realizado um levantamento dos profissionais de saúde segundo a categoria profissional, das áreas clínicas e de enfermagem e suas respectivas escalas de trabalho. Durante a pesquisa, foram percorridos os setores do hospital, segundo as escalas de trabalho, nos quais os trabalhadores foram convidados a participar da pesquisa e, posteriormente, foram aplicados os questionários. A exclusão do grupo amostral deu-se após três tentativas infrutíferas e, neste caso, o profissional era substituído pelo próximo participante da lista sorteada.

Os seguintes grupos de variáveis foram analisados. Variáveis sociodemográficas: sexo (masculino e feminino); idade em anos completos e categorizadas em quatro faixas etárias ( $\leq 30$ anos, 31 a 40 anos, 41 a 50 anos e $\geq 50$ anos); escolaridade (até o ensino médio e superior); situação conjugal (solteiro, casado/união estável e separado/viúvo; raça/ cor (branca/amarela e preta/parda/indígena); existência de filhos (sim e não); residência em Jequié (sim e não). Variáveis ocupacionais: tempo de serviço na profissão ( $\leq 5$ anos, 6 a 10 anos e $\geq 11$ anos); anos de trabalho no HGPV ( $\leq 5$ anos, 6 a 10 anos e $\geq 11$ anos); carga horária semanal total (20 horas, 30 horas e 40 horas); renda total (até $1 \mathrm{SM}$, até $2 \mathrm{SM}$ e $3 \mathrm{SM}$ ou mais); outra atividade remunerada (sim e não); aspectos psicossociais do trabalho (alta exigência, trabalho ativo, baixa 
exigência e trabalho passivo); realização de trabalho doméstico (sim e não). Variáveis sobre condições de saúde: estado de saúde autodeclarado (muito bom/bom, regular e ruim/muito ruim); tabagismo atual (sim e não); consumo de bebidas alcoólicas (sim e não); prática de atividade física (suficientemente ativo e insuficientemente ativo); transtornos mentais comuns (sim e não); comorbidades (sim e não); existência de doenças com diagnóstico médico (nenhuma, uma, duas e três ou mais); principais doenças com diagnóstico; sintomas musculoesqueléticos (geral, membros inferiores, membros superiores e dorso). Inicialmente, foi realizada uma análise descritiva das variáveis de interesse. Para tanto, foram utilizadas frequências simples e relativas para as variáveis categóricas e estatísticas descritivas (média, mediana e desvio-padrão) para as variáveis numéricas. As análises foram estratificadas pela função do trabalhador em assistencial ou administrativa. O teste de qui-quadrado de Pearson foi utilizado para avaliar a diferença entre os grupos de função, o valor de probabilidade do teste menor ou igual a 0,05 foi utilizado para considerar tal medida estatisticamente significante. Todos os dados colhidos foram tabulados com o auxílio do software Epidata, versão 3.1 e analisados com auxílio do programa estatístico "Social Package for Statistical Sciences - SPSS”, versão 21.0 for Windows.

O presente estudo seguiu as recomendações em vigor referentes à pesquisa envolvendo seres humanos, conforme a Resolução n. 196/96, do Conselho Nacional de Saúde, vigente à época do estudo. Foi assegurado aos profissionais de saúde o sigilo dos dados fornecidos e confidencialidade das informações. Estas somente foram utilizadas para atender aos objetivos da pesquisa. O projeto foi aprovado pelo Comitê de Ética e Pesquisa (CEP) da Universidade Estadual do Sudoeste da Bahia - Campus de Jequié - BA, Protocolo número 094/2011; CAAE: 0073.0.454.000-11.

\section{RESULTADOS}

Participaram do estudo 212 profissionais de saúde do Hospital Geral Prado Valadares (HGPV) em Jequié-Bahia. Com relação às características sociodemográficas (Tabela 1) e levando-se em consideração a ocupação/função dos profissionais, dicotomizadas em assistencial e administrativa, tem-se uma maior frequência daquela sobre esta, com valores respectivos de $72 \%$ e $28 \%$.

Em ambos os setores, a maioria dos profissionais pertenciam ao sexo feminino, correspondendo a $76,4 \%$ no setor assistencial e $77,8 \%$ no administrativo. A média de idade foi de $38,4 \pm 10,6$ anos para os trabalhadores da área assistencial, enquanto na área administrativa, a média de idade foi ligeiramente maior, correspondendo a 40,9 $\pm 11,2$ anos; em ambas, a faixa etária de maior frequência se encontrava no intervalo entre 31 e 40 anos. Com relação à escolaridade, ocorreu um predomínio da formação até o ensino médio em ambos os setores, apresentando os respectivos valores de $53,8 \%$ e $55,4 \%$, para o setor assistencial e administrativo. Os casados ou em união estável se configuravam como maioria nos dois setores, correspondendo a $63 \%$ no setor assistencial e $61,8 \%$ no administrativo. Em ambas as áreas, a cor da pele autodeclarada, incluída na categoria preta/ 
Tabela 1. Caracterização dos profissionais de saúde hospitalar segundo variáveis sociodemográficas

\begin{tabular}{|c|c|c|c|c|c|c|}
\hline \multirow{3}{*}{ Variáveis } & \multicolumn{6}{|c|}{ Ocupação / função } \\
\hline & \multicolumn{2}{|c|}{ Total } & \multicolumn{2}{|c|}{ Assistencial } & \multicolumn{2}{|c|}{ Administrativa } \\
\hline & $\mathrm{n}$ & $\%$ & $\mathrm{n}$ & $\%$ & $n$ & $\%$ \\
\hline \multicolumn{7}{|l|}{ Sexo* } \\
\hline Masculino & 48 & 23,6 & 35 & 23,6 & 12 & 22,2 \\
\hline Feminino & 155 & 76,4 & 113 & 76,4 & 42 & 77,8 \\
\hline \multicolumn{7}{|l|}{ Idade* } \\
\hline$\leq 30$ anos & 45 & 26,8 & 37 & 30,3 & 08 & 17,8 \\
\hline 31 a 40 anos & 59 & 35,1 & 42 & 34,4 & 17 & 37,8 \\
\hline 41 a 50 anos & 33 & 19,6 & 22 & 18,0 & 10 & 22,2 \\
\hline$\geq 50$ anos & 31 & 18,5 & 21 & 17,2 & 10 & 22,2 \\
\hline \multicolumn{7}{|l|}{ Escolaridade* } \\
\hline Até Ensino Médio & 110 & 54,5 & 78 & 53,8 & 31 & 55,4 \\
\hline Superior & 92 & 45,5 & 67 & 46,2 & 25 & 44,6 \\
\hline \multicolumn{7}{|l|}{ Situação conjugal* } \\
\hline Solteiro & 56 & 27,7 & 38 & 26,0 & 17 & 30,9 \\
\hline Casado / União estável & 126 & 62,4 & 92 & 63,0 & 34 & 61,8 \\
\hline Separado / Viúvo & 20 & 9,9 & 16 & 11,0 & 04 & 7,3 \\
\hline \multicolumn{7}{|l|}{ Raça / Cor* } \\
\hline Branca/ Amarela & 60 & 30,2 & 41 & 28,5 & 19 & 35,2 \\
\hline Preta/ Parda/ Indígena & 139 & 69,8 & 103 & 71,5 & 35 & 64,8 \\
\hline \multicolumn{7}{|l|}{ Filhos $^{* *}$} \\
\hline Sim & 131 & 71,6 & 93 & 70,5 & 37 & 74,0 \\
\hline Não & 52 & 28,4 & 39 & 29,5 & 13 & 26,0 \\
\hline \multicolumn{7}{|l|}{ Reside em Jequié ex* $^{* *}$} \\
\hline Sim & 175 & 85,4 & 121 & 81,2 & 53 & 96,4 \\
\hline Não & 30 & $\overline{14,6}$ & 28 & 18,8 & 02 & 3,6 \\
\hline
\end{tabular}

*sem significância estatística; ${ }^{* *} p<0,05^{* * *} p<0,01$

parda/indígena, apresentou maior frequência, equivalendo a $71,5 \%$ na área assistencial e $64,8 \%$ na administrativa. Nos dois setores, a maioria dos trabalhadores relataram a presença de filhos, equiparando-se em ambos, apresentando os valores de $70,5 \%$ no setor assistencial e $74 \%$ no administrativo. Com relação à residência na Cidade de Jequié, a frequência foi elevada em ambos os grupos de trabalhadores, cujos valores corresponderam a $81,2 \%$ no setor assistencial e $96,4 \%$ no setor administrativo. As variáveis presença de filhos e residência em Jequié apresentaram significância estatística. 
A Tabela 2 apresenta informações acerca das características ocupacionais desses trabalhadores. A maior parte dos profis- sionais $(48,1 \%)$, independentemente do tipo de ocupação, exerce a sua profissão há um período de tempo igual ou superior a 11 anos.

Tabela 2. Caracterização dos profissionais de saúde hospitalar segundo variáveis ocupacionais

\begin{tabular}{|c|c|c|c|c|c|c|}
\hline \multirow{3}{*}{ Variáveis } & \multicolumn{6}{|c|}{ Ocupação / função } \\
\hline & \multicolumn{2}{|c|}{ Total } & \multicolumn{2}{|c|}{ Assistencial } & \multicolumn{2}{|c|}{ Administrativa } \\
\hline & $\mathrm{n}$ & $\%$ & $\mathrm{n}$ & $\%$ & $\mathrm{n}$ & $\%$ \\
\hline \multicolumn{7}{|l|}{$\begin{array}{l}\text { Tempo de serviço na } \\
\text { profissão* }\end{array}$} \\
\hline$\leq 5$ anos & 56 & 26,4 & 44 & 32,6 & 12 & 22,6 \\
\hline 6 a 10 anos & 42 & 22,2 & 30 & 22,2 & 12 & 22,6 \\
\hline$\geq 11$ anos & 91 & 48,1 & 61 & 45,2 & 29 & 54,7 \\
\hline \multicolumn{7}{|l|}{$\begin{array}{l}\text { Anos de trabalho no } \\
\text { HGPV** }\end{array}$} \\
\hline$\leq 5$ anos & 90 & 55,6 & 68 & 61,3 & 22 & 44,0 \\
\hline 6 a 10 anos & 11 & 6,8 & 09 & 8,1 & 02 & 4,0 \\
\hline$\geq 11$ anos & 61 & 37,7 & 34 & 30,6 & 26 & 52,0 \\
\hline \multicolumn{7}{|c|}{$\begin{array}{l}\text { Carga horária semanal } \\
\text { total }^{* * *}\end{array}$} \\
\hline 20 horas & 005 & 2,8 & 04 & 3,3 & 01 & 1,7 \\
\hline 30 horas & 98 & 54,7 & 76 & 63,3 & 22 & 37,9 \\
\hline 40 horas & 76 & 42,5 & 40 & 33,3 & 35 & 60,3 \\
\hline \multicolumn{7}{|l|}{ Renda total $\left.\right|^{(\mathrm{a}) *}$} \\
\hline Até $1 \mathrm{SM}$ & 11 & 7,4 & 06 & 5,7 & 05 & 11,6 \\
\hline Até 2 SM & 34 & 22,8 & 22 & 21,0 & 12 & 27,9 \\
\hline $3 \mathrm{SM}$ ou mais & 104 & 69,8 & 77 & 73,3 & 26 & 60,5 \\
\hline \multicolumn{7}{|l|}{$\begin{array}{l}\text { Outra atividade } \\
\text { remunerada* }\end{array}$} \\
\hline Sim & 89 & 43,4 & 70 & 46,7 & 18 & 33,3 \\
\hline Não & 116 & 56,6 & 80 & 53,3 & 36 & 66,7 \\
\hline \multicolumn{7}{|c|}{$\begin{array}{l}\text { Aspectos psicossociais do } \\
\text { trabalho* }\end{array}$} \\
\hline Alta exigência & 34 & 22,7 & 25 & 23,1 & 08 & 19,5 \\
\hline Trabalho ativo & 42 & 28,0 & 33 & 30,6 & 09 & 22,0 \\
\hline Baixa exigência & 42 & 28,0 & 31 & 28,7 & 11 & 26,8 \\
\hline Trabalho passivo & 32 & 21,3 & 19 & 17,6 & 13 & 31,7 \\
\hline \multicolumn{7}{|l|}{ Trabalho doméstico* } \\
\hline Sim & 170 & 83,7 & 122 & 83,6 & 48 & 85,7 \\
\hline Não & 33 & 16,3 & 24 & 16,4 & 08 & 14,3 \\
\hline
\end{tabular}

(a) SM-Salário mínimo.

*sem significância estatística; ${ }^{* *} p<0,05^{* * *} p<0,01$ 
Levando-se em consideração somente o tipo de ocupação/trabalho, notou-se que $61,3 \%$ dos trabalhadores da função assistencial trabalham no local há um período menor ou igual a 5 anos e $52,0 \%$ dos profissionais trabalham 11 anos ou mais.

Além disso, tem-se que o regime de trabalho majoritário no hospital foi o de 30 horas semanais $(54,7 \%)$. Os profissionais do setor assistencial corroboram esse dado ao apresentar $63,3 \%$ de seus profissionais com essa carga horária semanal; enquanto no setor administrativo, a maioria $(60,3 \%)$ dos trabalhadores tem 40 horas semanais de trabalho no HGPV. Ambos os grupos têm renda total com um valor igual ou superior à três salários mínimos, apresentando 69,8\% como porcentagem total.

Do total de trabalhadores, $43,4 \%$ exerciam alguma outra atividade remunerada; dentro dessa variável, percebeu-se um predomínio dos profissionais que desempenham funções assistenciais $(46,7 \%)$, sobre aqueles que desempenham funções administrativas (33,3\%). Considerando-se os aspectos psicossociais do trabalho, ocorreu uma distribuição bastante equilibrada entre as categorias, com destaque para o trabalho ativo (28\%), que, inclusive, foi mais preponderante dentre os trabalhadores de funções assistenciais $(30,6 \%)$. Levando-se em consideração a atividade de trabalho doméstico, ocorreu uma expressiva porcentagem $(83,7 \%)$ de trabalhadores, que, independentemente do tipo de função/ocupação, a realiza.

Com relação ao estado de saúde autodeclarado (Tabela 3), a maioria dos participantes o considera como muito bom/bom $(80,5 \%)$, sendo os do sexo masculino os mais satisfeitos (87,5\%); ainda neste quesito, os profissionais da área assistencial e administrativa não demonstraram grandes diferenças no que concerne a boa avaliação do estado de saúde, apresentando, respectivamente, os valores de $83 \%$ e $73,7 \%$.

Levando-se em consideração os hábitos de vida que podem influenciar na saúde dos trabalhadores, o hábito tabágico apresentou porcentagem total de 3,9\%; o consumo de álcool, apresentou porcentagem total de $37,9 \%$, não existindo grandes diferenças percentuais entre o tipo de ocupação dos trabalhadores; no entanto, a insuficiência da prática de atividade física apresentou valor expressivo total de $90,1 \%$.

Ainda referente a descrição de saúde dos trabalhadores do estudo, $74,1 \%$ dos trabalhadores apresentavam alguma comorbidade (Tabela 3). Analisando-se a existência de doenças com diagnóstico médico prévio, tem-se a categoria três ou mais com destaque em ambos os grupos de profissionais, com os valores de $28,4 \%$ e $11,3 \%$ para os trabalhadores do setor assistencial e administrativo, respectivamente. Nesse grupo de doenças, as mais prevalentes foram: lombalgia $(36,1 \%)$, rinite/sinusite $(35 \%)$, varizes $(28,4 \%)$, gastrite $(26,5 \%)$, colesterol alto $(23,3 \%)$, hipertensão (23\%), hérnia de disco (19\%) e distúrbios do sono $(17,5 \%)$. Os transtornos mentais comuns nesse grupo corresponderam a $25,8 \%$, sendo mais prevalente nos trabalhadores do setor administrativo do hospital (44,9\%).

Os sintomas osteomusculares se mostraram bastante presentes na caracterização de saúde desses profissionais. De modo geral, 157 profissionais $(78,1 \%)$ relataram algum sintoma musculoesquelético. Segmentando- 
Tabela 3. Caracterização dos profissionais de saúde hospitalar segundo as condições de saúde

\begin{tabular}{|c|c|c|c|c|c|c|}
\hline \multirow{3}{*}{ Variáveis } & \multicolumn{6}{|c|}{ Ocupação / função } \\
\hline & \multicolumn{2}{|c|}{ Total } & \multicolumn{2}{|c|}{ Assistencial } & \multicolumn{2}{|c|}{ Administrativa } \\
\hline & $\mathrm{n}$ & $\%$ & $\mathrm{n}$ & $\%$ & $\mathrm{n}$ & $\%$ \\
\hline \multicolumn{7}{|c|}{ Estado de saúde autodeclarado* } \\
\hline Muito bom/ Bom & 165 & 80,5 & 122 & 83,0 & 42 & 73,7 \\
\hline Regular & 36 & 17,6 & 22 & 15,0 & 14 & 24,6 \\
\hline Ruim/ Muito ruim & 04 & 2,0 & 03 & 2,0 & 01 & 1,8 \\
\hline \multicolumn{7}{|l|}{ Tabagismo atual* } \\
\hline Sim & 08 & 3,9 & 04 & 2,7 & 04 & 6,9 \\
\hline Não & 197 & 96,1 & 142 & 97,3 & 54 & 93,1 \\
\hline \multicolumn{7}{|c|}{ Consumo de bebidas alcoólicas* } \\
\hline Sim & 77 & 37,9 & 54 & 37,0 & 23 & 41,1 \\
\hline Não & 126 & 62,1 & 92 & 63,0 & 33 & 58,9 \\
\hline \multicolumn{7}{|l|}{ Prática de atividade física* } \\
\hline Suficientemente ativo & 14 & 9,9 & 12 & 11,4 & 02 & 5,7 \\
\hline Insuficientemente ativo & 127 & 90,1 & 93 & 88,6 & 33 & 94,3 \\
\hline \multicolumn{7}{|c|}{ Transtornos mentais comuns ${ }^{\star * *}$} \\
\hline Sim & 47 & 25,8 & 25 & 18,9 & 22 & 44,9 \\
\hline Não & 135 & 74,2 & 107 & 81,1 & 27 & 55,1 \\
\hline \multicolumn{7}{|l|}{ Comorbidades* $^{*}$} \\
\hline Sim & 157 & 74,1 & 111 & 73,0 & 45 & 76,3 \\
\hline Não & 55 & 25,9 & 41 & 27,0 & 14 & 23,7 \\
\hline \multicolumn{7}{|c|}{ Doenças com diagnóstico médico* } \\
\hline Nenhuma & 55 & 25,9 & 41 & 19,4 & 14 & 6,6 \\
\hline Uma & 37 & 17,4 & 24 & 11,3 & 12 & 5,6 \\
\hline Duas & 36 & 16,9 & 27 & 12,8 & 09 & 4,2 \\
\hline Três ou mais & 84 & 39,6 & 60 & 28,4 & 24 & 11,3 \\
\hline \multicolumn{7}{|c|}{ Principais doenças com diagnóstico* } \\
\hline Lombalgia & 65 & 36,1 & 47 & 35,6 & 18 & 38,3 \\
\hline Rinite/sinusite & 63 & 35,0 & 47 & 35,1 & 16 & 35,6 \\
\hline Varizes & 50 & 28,4 & 37 & 28,1 & 13 & 28,9 \\
\hline Gastrite & 48 & 26,5 & 33 & 24,6 & 15 & 32,6 \\
\hline Colesterol alto & 42 & 23,3 & 31 & 23,1 & 11 & 24,4 \\
\hline Hipertensão & 42 & 23,0 & 27 & 20,1 & 15 & 31,3 \\
\hline Hérnia de disco & 34 & 19,0 & 24 & 18,3 & 10 & 21,3 \\
\hline Distúrbios do sono & 31 & 17,5 & 20 & 15,4 & 11 & 23,9 \\
\hline \multicolumn{7}{|c|}{ Sintomas musculoesqueléticos ${ }^{*}$} \\
\hline Geral & 157 & 78,1 & 109 & 75,7 & 47 & 83,9 \\
\hline Membros inferiores & 101 & 52,6 & 68 & 49,3 & 33 & 62,3 \\
\hline Membros superiores & 111 & 57,5 & 79 & 56,0 & 32 & 62,7 \\
\hline Dorso & 126 & 65,3 & 95 & 66,9 & 30 & 60,0 \\
\hline
\end{tabular}

*sem significância estatística; ${ }^{* *} p<0,05^{* * *} p<0,01$ 
-se por regiões corporais: $57,5 \%$ apresentam sintomas nos membros superiores, $52,6 \%$ nos membros inferiores e $65,3 \%$ apresentam sintomas osteomusculares no dorso. Com relação à ocupação, os profissionais do setor administrativo apresentaram um percentual maior de frequência de sintomas musculoesqueléticos (83,9\%), quando comparados aos do setor assistencial $(75,7 \%)$. Verificando-se por regiões corporais, percebeu-se que a frequência dos sintomas em membros inferiores e superiores permaneceu maior nos trabalhadores da área assistencial, entretanto, quando compara-se a região dorsal nota-se um valor ligeiramente maior nessa área (66,9\%), em comparação com aquela $(60 \%)$.

\section{DISCUSSÃO}

O presente estudo permitiu a descrição do perfil sociodemográfico e das condições gerais de saúde dos profissionais que trabaIhavam no HGPV em Jequié (BA), segundo o perfil de trabalho.

O trabalho em saúde, mais propriamente, aquele desempenhado no âmbito hospitalar é caracterizado por alta demanda, física e/ou psicológica e isso se reflete na saúde de seus trabalhadores. O regime de trabalho, muitas vezes, baseado em plantões e em longas jornadas, além de uma necessidade de alta produtividade e respostas rápidas às ocorrências, dentre muitos outros fatores, colaboram para o adoecimento desses trabaIhadores ${ }^{9,10}$.

Após a avaliação dos dados obtidos, pode-se considerar que o trabalho em saúde, no HGPV, tem sido realizado predominantemente por mulheres, adultas jovens, grande parte casada e com filhos. O predomínio do sexo feminino no trabalho em saúde tem raízes na divisão sexual do trabalho, no qual, o cuidado inicialmente com a casa e as crianças, com a inserção feminina no mercado de trabalho, se expandiu para o cuidado com os outros $^{11,12}$.

Levando-se em consideração a carga horária semanal dos trabalhadores no HGPV, percebe-se que, dentre os profissionais de função assistencial, a maioria corresponde a 30h, enquanto os de ocupação administrativa apresentam, majoritariamente, a carga horária semanal de 40h. Isso pode justificar, inclusive, o fato de os profissionais do setor assistencial desempenharem, mais frequentemente outra atividade remunerada. Estudo realizado com profissionais médicos no Estado do Ceará ${ }^{13}$ apontou que a carga de trabalho desempenhada por estes trabalhadores variava de 20 a 70 horas semanais, considerando-se apenas os vínculos no setor público. Outro estudo ${ }^{14}$, realizado na Bahia, com a população de enfermeiros, identificou uma porcentagem que variava entre $57,8 \%$ e $73,3 \%$, a depender do tipo de contrato de trabalho, de profissionais que tinham dois vínculos empregatícios.

Além disso, deve ser considerado o trabalho desempenhado em ambiente doméstico, que se configura como uma sobrecarga a esse trabalhador, devido à dupla exposição. Em estudo realizado com uma população de enfermeiros, as mulheres apresentaram a sua jornada de trabalho aumentada, em comparação aos homens, pois, apesar de também estarem inseridas no mercado formal de trabaIho, elas ainda continuam sendo as principais responsáveis pelas atividades domésticas ${ }^{15}$. Entretanto, tal estudo ressalta que o trabalho 
doméstico pode ser visto também como prazeroso, visto que pode contribuir para uma maior proximidade do trabalhador com sua família.

A autopercepção acerca do estado de saúde demonstrou um alto grau de satisfação dos profissionais estudados com sua saúde, quando comparada a outros de mesma idade. Esse achado se mostrou superior ao encontrado em um estudo realizado em um Hospital Universitário em Londrina ${ }^{16}$, no qual a porcentagem de satisfação encontrada no sexo feminino foi de $53,2 \%$ e no masculino de $75 \%$, comparados aos encontrados no presente estudo. Não houve discordância, entretanto, com relação ao sexo; o sexo feminino desponta como mais insatisfeito.

Analisando os aspectos psicossociais do trabalho, de acordo com o modelo demanda-controle (MDC), proposto por Karasek ${ }^{17}$, percebeu-se uma distribuição ligeiramente equilibrada entre as categorias. É importante que tais aspectos sejam analisados devido ao fato de influenciarem a saúde do trabalhador, principalmente, em nível psíquico, e, por conseguinte, o seu desempenho laboral. Estudo realizado entre enfermeiros de um grupo hospitalar em Porto Alegre/RS ${ }^{18}$, constatou maior prevalência das categorias trabalho ativo $(43,2 \%)$ e baixa exigência $(20,7 \%)$, em consonância com os $28 \%$ encontrados em ambas as categorias no presente estudo, quando foi analisado o perfil geral dos trabalhadores. Quando se observa o MDC em comparação com os setores assistencial e administrativo, visualizou-se uma prevalência aumentada das categorias de alta exigência, trabalho ativo e baixa exigência no grupo de trabalhadores assistenciais, enquanto o trabalho passivo foi mais prevalente entre os profissionais do setor administrativo.
Com relação às doenças crônicas já diagnosticadas à época da aplicação do questionário, destacou-se a lombalgia, que é considerada a doença ocupacional mais antiga relatada pela história ${ }^{19}$, além de ser apontada como a maior causa de transtorno de saúde associado ao trabalho e de absenteísmo ${ }^{20}$. Considerando-se o trabalhador da saúde, estudos ${ }^{21,22} \mathrm{com}$ a população de enfermeiros que trabalhavam em hospitais, apontaram a grande prevalência de tal evento, principalmente, quando associado às condições de trabalho não ideais.

Ainda nessa temática, destacou-se a alta prevalência de sintomas osteomusculares nos profissionais analisados, fato este que tem respaldo na literatura, principalmente, no que diz respeito aos trabalhadores de enfermagem. Além dos sintomas relacionados ao dorso, são comuns as queixas relacionadas aos membros superiores e inferiores, como lesão por esforço repetitivo (LER) e a sensação de peso e cansaço nas pernas, respectivamente $^{23}$. Apesar de não ter sido analisado os sintomas de dor musculoesquelética por categorias profissionais, os achados desta pesquisa chamaram a atenção para uma elevada prevalência desse evento para as duas funções de trabalho investigadas.

Destacou-se, por fim, a elevada prevalência de trabalhadores que não praticam atividade física regular, configurando-se como insuficientemente ativos. Sabe-se que o sedentarismo é um dos maiores fatores de risco para o desenvolvimento de doenças crônicas não transmissíveis, tal fator, inclusive, pode estar relacionado à alta incidência de sintomas osteomusculares nessa população. Estudos recentes $^{16,24}$ corroboram com esse achado 
e, apesar de apresentarem porcentagens relativamente menores, apontaram para a tendência de negligência da prática de atividade física por parte dos trabalhadores da saúde.

O presente estudo obteve um conjunto de resultados importantes, que têm a possibilidade de contribuir para a melhora da compreensão da situação de saúde do trabalhador do setor de saúde hospitalar. Entretanto, possui algumas limitações como o fato de ser um estudo de corte transversal, analisando, ao mesmo tempo, causa e efeito, não sendo possível avaliar as relações diretas entre as variáveis; além disso, existe o efeito do trabalhador sadio, viés comum nos estudos em saúde do trabalhador, que, pelo fato de os trabalhadores estarem afastados do serviço, por motivo de agravos à saúde, acabam não sendo estatisticamente considerados. Não foi possível também verificar se os profissionais que exerciam função assistencial migraram para função administrativa motivados por suas condições de saúde e, nesta situação, os valores percentuais se aproximaram para os fatores investigados nesta pesquisa.

\section{CONCLUSÃO}

A análise das condições gerais de saúde dos trabalhadores da saúde hospitalar, possibilitou um melhor conhecimento de tal grupo, que se configura como majoritariamente feminino, na faixa etária de 31 a 40 anos, residentes na cidade onde trabalham, que realizam trabalho doméstico, com prática insuficiente de atividade física, presença de três ou mais doenças com diagnóstico médico, com sintomas musculoesqueléticos bastante prevalentes, entre outros achados, o que pode culminar em posterior direcionamento de cuidado à essa população, levando-se em consideração todos os fatores ocupacionais, que podem se configurar como de risco para o desenvolvimento de doenças relacionadas ao trabalho.

\section{REFERÊNCIAS}

1. Brasil. Poder Legislativo. Lei Orgânica da Saúde n.8.080, de 19 de setembro de 1990. Dispõe sobre as condições para a promoção, proteção e recuperação da saúde, a organização e o funcionamento dos serviços correspondentes e dá outras providências. Diário Oficial da República Federativa do Brasil. Brasília, DF, Seção 1, p.18.055, 1990.

2. Brasil. Ministério da Saúde. Secretaria de Políticas de Saúde. Departamento de Atenção Básica. Área Técnica de Saúde do Trabalhador. Saúde do Trabalhador. (Cadernos de Atenção Básica. Programa Saúde da Família; 5) Brasília: Ministério da Saúde; 2001.

3. Organização Internacional do Trabalho. A prevenção das doenças profissionais. 2013.

4. Nescon - Núcleo de Educação em Saúde Coletiva. Condições de trabalho e saúde dos trabalhadores da saúde / organizado por Ada Ávila Assunção e Soraya Almeida Belisário. Belo Horizonte: Nescon; 2007.

5. IBGE. População estimada da cidade de Jequié - Bahia. Instituto Brasileira de Geografia e Estatística; 2017.

6. Governo do Estado da Bahia. Hospital Geral Prado Valadares. 2019.

7. Ferracini GN, Valente FM. Presença de sintomas musculoesqueléticos e efeitos da ginástica laboral em funcionários do setor administrativo de um hospital público. Rev Dor. 2010; 1(3):233-236.

8. Christensen JR1, Faber A, Ekner D, Overgaard K, Holtermann A, Søgaard K. Diet, physical exercise and cognitive behavioral training as a combined workplace based intervention to reduce body weight and increase physical capacity in health care workers - a randomized controlled trial. BMC Public Health. 2011; 27(11):671.

9. Machado LSF, Rodrigues EP, Oliveira LMM, Laudano RCS, Nascimento SCL. Agravos à saúde referidos pelos trabalhadores de enfermagem em um hospital público da Bahia. Rev. Bras. Enferm. 2014; 67(5):684-691. 
10. Santana LL, Sarquis LMM, Miranda FMD, Kalinke LP, Felli VEA, Mininel VA. Indicadores de saúde dos trabalhadores da área hospitalar. Rev. Bras. Enferm. 2016; 69(1):30-39.

11. Gomes DJ, Araújo TM, Santos KOB. Condições de trabalho e de saúde de trabalhadores em saúde mental em Feira de Santana, Bahia. Rev. Baiana de Saúde Pública. 2011; 35 (supl.1):211-230.

12. Machado $\mathrm{MH}$, Wermelinger $\mathrm{M}$, Tavares MFL, Moysés NMN, Teixeira M, Oliveira ES. Análise da força de trabalho do setor saúde no Brasil: focalizando a feminização. Rio de Janeiro: Fundação Oswaldo Cruz, Rede Observatório de Recursos Humanos em Saúde; 2006.

13. Maciel RH, Santos JBF, Sales TB, Alves MAA, Luna AP, Feitosa LB. Multiplicidade de vínculos de médicos no Estado do Ceará. Rev. Saúde Pública. 2010; 44(5):950956.

14. Zatti MLR, Mendes VLPS. Comprometimento organizacional na área hospitalar e vínculos empregatícios. XXXI Encontro da ANPAD. Rio de Janeiro/RJ. 2007.

15. Fernandes JC, Portela LF, Rotenberg L, Griep RH. Jornada de trabalho e comportamentos de saúde entre enfermeiros de hospitais públicos. Rev. Latino-Am. Enfermagem. 2013; 21(5):1-7.

16. Porto DB, Arruda GA, Altimari LR, Cardoso Júnior CG. Autopercepção de saúde em trabalhadores de um Hospital Universitário e sua associação com indicadores de adiposidade, pressão arterial e prática de atividade física. Ciênc. Saúde coletiva [Internet]. 2016; 21(4):1113-1122.

17. Karasek R. Job Demand, job decision latitude, and mental strain: implications for job redesign. Admin Sci Quar. 1979; 24:285-308.
18. Negeliskii C, Lautert L. Estresse laboral e capacidade para o trabalho de enfermeiros de um grupo hospitalar. Rev. Latin-Am. Enfermagem. 201; 19(3):606-613.

19. Dias EC, Godoy SCB, Almeida V. Desafio da abordagem multidisciplinar da lombalgia ocupacional. Rev. Min. Enf. 2003; 7(1):67-72.

20. Helfenstein Júnior M, Goldenfum MA, Siena C. Lombalgia ocupacional. Rev. Assoc. Med. Bras. 2010; 56(5):583-589.

21. Borges TP, Kurebayashi LFS, Silva MJP. Lombalgia ocupacional em trabalhadores de enfermagem: massagem versus dor. Rev. Esc. Enferm. USP. 2014; 48(4):670-676.

22. Serranheira F, Sousa-Uva M, Sousa-Uva A. Lombalgias e trabalho hospitalar em enfermeiro(a)s. Rev. Bras. Med. Trab. 2012; 10(2):80-87.

23. Souza AC, Coluci MZO, Alexandre NMC. Sintomas osteomusculares em trabalhadores da enfermagem: uma revisão integrativa. Cienc. Cuid.Saude. 2009; 8(4):683690.

24. Sousa PTM, Sousa ARR, Pacheco ES, Sousa GTM. Comportamento sedentário entre profissionais da Estratégia de Saúde da Família. Rev. Enferm. 2017; 6(3):24-29.

\section{CORRESPONDÊNCIA}

Jefferson Paixão Cardoso,

Universidade Estadual do Sudoeste da Bahia,

Departamento de Saúde II,

Avenida José Moreira Sobrinho sn, Jequiezinho,

Jequié-Bahia-Brasil, CEP 45208-409.

E-mail: jpcardoso@uesb.edu.br 